\title{
Problematic Smartphone Use in a Large Nationally Representative Sample: Age, Reporting Biases, and Technology Concerns
}

\author{
Sharon Horwood, Jeromy Anglim, Sumudu R. Mallawaarachchi ${ }^{1}$
}

\begin{abstract}
This study utilized data from a nationally representative sample of Australian adults ( $n=1164 ; 50.7 \%$ female; age $M=44.9$ years, $S D=16.3)$ to examine the relationships between age, technology concerns, self-rated and objective amount of smartphone use, and problematic smartphone use. Participants completed measures of problematic smartphone use and technology concern, while amount of smartphone use was self-rated and objectively measured using smartphone screen time reporting tools (Screen Time for iOS and Digital Wellbeing for Android). Amount of self-rated and objective smartphone use declined linearly with age. In contrast, problematic smartphone use was relatively high and stable in young adults before rapidly declining around age 40. People were reasonably good at estimating their amount of smartphone use $(r=.73)$, although they did tend to underestimate usage. Technology concern was high across all ages, but unrelated to amount of usage and problematic smartphone usage. Age related differences are interpreted in terms of a combination of developmental and generational changes. Results also suggest that amount of use is an important but not complete cause of problematic smartphone use.
\end{abstract}

Keywords: age, problematic smartphone use, smartphone addiction, objective smartphone use, fear of technology

\section{Introduction}

The introduction of new technology has often been accompanied by concerns about imminent detriments to society generally, and to young users specifically. Past concerns have included fears that new technologies such as television and video games lead young people to become desensitized to aggression (Belson \& Belson, 1978; Carnagey, Anderson, \& Bushman, 2007; Kirsh, 2003; Kremar \& Greene, 1999), increasingly sedentary (Biddle, Gorely, \& Marshall, 2009; Ford et al., 2010; Rey-López, Vicente-Rodríguez, Biosca, \& Moreno, 2008), and socially isolated (Bickham \& Rich, 2006; Stern, 1999). There is now an emerging debate about whether smartphones are harming young people or whether this belief is simply the latest manifestation of a tendency for society to fear new technologies (Anderson \& Rainie, 2018; Orben, 2020). Exploring the effect of age on problematic

\section{Citation:}

Horwood, S., Anglim, J. \& Mallawaarachchi, S. (2021). Problematic Smartphone Use in a Large Nationally Representative Sample: Age, Reporting Biases, and Technology Concerns.

Computers in Human Behavior, 106848. https://doi.org/10.1016/j.chb.2021

School of Psychology, Deakin University, Geelong, Australia Data, analysis scripts, and study materials are available on the OSF at https://osf.io/cdbzg Correspondence concerning this article should be addressed to Sharon Horwood, School of Psychology, Deakin University, Locked Bag 20000, Geelong, 3220 Australia. Email: sharon.horwood@deakin.edu.au 
smartphone use may help to establish whether smartphone usage could contribute to negative health behavior patterns and habits in young adulthood, behaviors which could have profound effects on life-long health and wellbeing and bring with them major social and economic impacts.

Despite the growing literature on problematic smartphone use several important theoretical and methodological gaps remain. First, limited research has examined whether the effect of age on smartphone use is non-linear. Most studies have used convenience samples of young adults, principally university undergraduate students, which impedes a full examination of the association between age and smartphone use. Second, little research has considered whether the effect of age on amount of use is the same or different to problematic use. Third, it is not clear whether self-rated smartphone use is a reliable measure of actual (objective) smartphone use. Most studies have relied on self-report inventories to measure amount of smartphone use, and the small number of studies that have measured objective use have found that predictions of self-rated use are moderately effective ( $\mathrm{r}=.38$, Parry et al., 2020). Fourth, no studies to date have related problematic smartphone use to technology concerns in order to assess the degree to which problematic smartphone use reflects a general concern with new technology.

\subsection{Problematic Smartphone Use and Amount of Smartphone Use}

Problematic smartphone use can be defined as compulsive use that leads to impaired daily functioning in terms of productivity, social relationships, physical health, or emotional well-being (Horwood \& Anglim, 2018). It is a complex construct, and likely caused by more than just total amount of use. Different people seem to appraise the same type or amount of use as more or less problematic, and people who have the same amount of objective use can differ in their subjective experiences, such as anxiety or fear of missing out, when they are away from their smartphone. The relative importance of amount of use, affective experience, or generalized perceptions of smartphone use as harmful on appraisals of problematic smartphone use remain unclear, however several studies have examined the association between problematic smartphone use and amount of use (e.g., Lee, Han, \& Pak, 2018; Pan, Lin, Chiu, Lin, \& Lin, 2019; Prasad et al., 2018; Rozgonjuk, Levine, Hall, \& Elhai, 2018). In a systematic review of the literature, Ryding and Kuss (2020) identified 18 studies that used some form of objective measurement of smartphone use to assess the association between objective use and problematic use. The review found that as the amount of use increased, so did problematic use, however all of the studies in the review relied on small convenience samples which makes extrapolation and broad inferences about usage difficult. Other studies have examined the degree to which people can accurately estimate the amount of time they spend on their smartphone (e.g., Wilcockson, Ellis, \& Shaw, 2018). For instance, Ellis, Davidson, Shaw, and Geyer (2019) examined a sample of university students and found that objective and self-rated usage time was correlated $r=.48$. They found that objective use per day $(M=233$ minutes, $S D=119)$ was similar but slightly higher than the self-rated use $(M=227$ minutes, $S D=128)$. A recent systematic review and meta-analysis of self-rated versus objectively measured smartphone use reported a moderate overall correlation $(\mathrm{r}=.38$, Parry et al., 2020). Overall, this suggests that people have a reasonable sense of how much they use 
their smartphone but that such an impression is far from perfect. It is also likely that the correlation is attenuated in university samples because the variance in amount of use is less than would be expected when looking at a representative community sample which is likely to include many older adults who may use their smartphone less.

\subsection{Age and Smartphone Use}

The question of whether younger people are more vulnerable to problematic use of new technologies is predicated on assumptions about the amount and types of technology use younger people engage in compared to older people. Beliefs that younger people more readily adopt new technology or are somehow more instinctively familiar with it than older adults do bear out in smartphone uptake and ownership rates. Although smartphones are no longer considered 'new technology', there are some distinct differences in the types of people who are more likely to own a smartphone. For example, in 2019, 96\% of 18 to 29-year old Americans owned a smartphone compared to $56 \%$ of Americans aged 65 or over (Pew Research, 2019). Similarly, younger people are also bigger users of social media platforms commonly accessed via smartphones, and appear to experience more problematic use of social media compared to older adults (Rozgonjuk, Sindermann, Elhai, \& Montag, 2020). Around $90 \%$ of 18-29 year old's use social media compared to around $40 \%$ of people aged 35 and over (Pew Research, 2019). While research suggests that older people report less problematic smartphone use (for a review see Busch \& McCarthy, 2020), most studies have relied on convenience samples of undergraduate students that are predominantly young adults (e.g. Mitchell \& Hussain, 2018; Van Deursen, Bolle, Hegner, \& Kommers, 2015).

One of the challenges in examining correlations between age and smartphone use is disentangling developmental age effects from generational cohort effects. Zhitomirsky-Geffet and Blau (2016) argue that more recent generations have had greater exposure to smartphones during key developmental periods (e.g. adolescence) where life-long habits are more likely to be formed. Similarly, the idea of digital nativity (Prensky, 2001; Wang, Sigerson, \& Cheng, 2019) suggests that young adults who have grown up in a world where there has always been the internet, social media, and smartphones are more likely to 'naturally' use smartphone technology compared to older generations (digital immigrants). Other researchers have taken a developmental perspective by highlighting how certain age groups may be more susceptible to developing problematic smartphone use based on their life stage (Busch \& McCarthy, 2020; Csibi, Griffiths, Demetrovics, \& Szabo, 2019; Hsieh et al., 2020). For example, between the ages of 18 to 30, people may be more likely to be socializing and engaging in romantic relationship formation that can be facilitated via smartphone applications (e.g. dating apps and communication platforms). Younger people may also be less encumbered with timeconsuming life-stage events that are more typical of middle-aged or older people, such as career or child-rearing pursuits. It is also important to distinguish between the effect of age on problematic smartphone use versus the effect of age on amount of use. In particular, it is possible that norms about what is 'appropriate use' differ between age groups, or that the types of use are more problematic relative to frequency. To date, however, no studies have examined age effects on general and 
problematic smartphone use with a large, broad cross-section of the adult population.

\subsection{Technology Concern}

Public concerns about the impact of technology on health and wellbeing are not new. Since the introduction of the radio, fears for how new technology might alter the way people, particularly young people, might experience the world have become routine (Anderson \& Rainie, 2018; Orben, 2020). What is not well understood however, is who is concerned about new technology and whether beliefs and attitudes about the 'dangers' of new technology influence behavior towards technology. Modern social fears about smartphones and related technology mainly revolve around ideas about collection of big data (Dewandre, 2020), impacts of screen use on children (Clement \& Miles, 2018; Montag \& Elhai, 2020), technology overuse (Montag \& Walla, 2016), and fears about technology impacting health and wellbeing (Alter, 2017). Despite a growing literature examining the mental health associations of excessive smartphone use, an emerging debate in the smartphone literature suggests that problematic smartphone use is simply a manifestation of cyclical or predictable generational technology concerns and not a real and distinct psychological construct (Orben, 2020). If this is the case, that problematic smartphone use is effectively predictable technology fears by another name, there should be a strong positive relationship between technology concerns and problematic smartphone use. Another way to conceptualize the same argument is to propose that as people engage more with their technology, they may become more cognizant of their increased use and consider the technology itself to be a problem. Similarly, we should also expect to see a greater incidence of technology concern in older adults, given the common perception that young people are those most feared for as being at risk of adverse effects of new technology.

\subsection{The Current Study}

The current study aimed to assess the effect of age on smartphone use, the degree to which people are able to accurately estimate their amount of smartphone use, and the degree to which problematic smartphone use could be explained by age, amount of smartphone use, and technology concern. To achieve these aims we conducted a large nationally representative survey of Australian adults which included measures of demographics, self-rated usage, objective usage, problematic smartphone usage, and general technology concerns. In examining the effect of age on the various usage measures, we also examined the degree to which the effect of age was non-linear (i.e., did it plateau at certain ages or was it curvilinear).

In general, we had the following expectations. First, based on previous research we expected age to be negatively correlated with each of self-rated use, objective use, and problematic smartphone use. Second, based on previous research we expected that people would be reasonably good at predicting their actual smartphone use, but with a tendency to underestimate. Finally, we also examined the strength of association between problematic smartphone use and technology concern. A strong correlation would support the claim that problematic smartphone use mostly reflects broad social concerns about technology. In contrast, we expected the correlation to be weak, on the assumption that problematic smartphone use 
reflects real concerns held by individuals grounded in actual smartphone usage patterns.

\section{Method}

Data, analysis scripts, and study materials are available on the OSF at https://osf.io/cdbzg

\subsection{Participants and Procedure}

Data collection was commissioned by an Australian television broadcaster (SBS Australia) to inform a documentary about technology use in society. Participants were recruited by market research company Dynata, whose participant database consists of over 700,000 Australians. The sample was designed to be representative of the Australian adult population who use a smartphone. Specifically, quotas were employed so as to mirror Australian demographics on gender, age (18 to 24,25 to 34,35 to 44,45 to 54,55 to 64, and 65+), and Australian States and territories (Vic, NSW, Tas, QLD, SA, WA, NT, ACT). Thus, all participants were aged 18 or over and used either an Android smartphone or iPhone. Participants completed the survey online answering questions about demographics, smartphone use, technology concerns, self-rated smartphone use, and objective smartphone use. Data collection commenced on 11th of June 2020 with over $90 \%$ of data collected by 16 th of June 2020; data collection was finalized on the 28th of June 2020.

The final cleaned sample $(n=1164)$ was $50.7 \%$ Female, $49.1 \%$ Male, and $0.3 \%$ Non-Binary, with a mean age of 44.9 years $(S D=16.3$; range: 18 to 93$)$. State of residence was 34\% NSW, 25\% VIC, 18\% QLD, 9\% WA, 8\% SA, 2\% TAS, $\%$ ACT, and $1 \%$ NT. Participants were 58\% iPhone users and $42 \%$ Android smartphone users. This final sample was drawn from an initial sample of 2000 participants. Participants were excluded if they did not provide complete data or there were signs that they had not completed all questions accurately. Participants were initially excluded because either they did not provide their objective smartphone use data $(n=636)$ or they did not provide a self-rated estimate of their smartphone usage data $(n=23)$. Participants were additionally excluded if they met any of the following conditions: (a) they only used one response option on the 18item survey $(n=40)$, (b) objective daily smartphone use was reported to be more than 12 hours per day $(\mathrm{n}=25)$, (c) self-rated daily smartphone use was less than 4 hours per day and objective use was reported as greater than 8 hours per day $(n=$ 42), and (d) for Android users, where objective usage was entered separately for each of 7 days, daily use had fewer than 4 unique time entries [i.e., entering identical hours and minutes on several different days] $(n=112)$. As a robustness check, results are shown in the online supplement using pairwise deletion for cases with one or missing variables $(n=1790)$. Results were not altered in any substantive manner.

The decision to source 2,000 participants from the market research company was made by SBS Australia with our guidance. This sample size sought to balance recruitment costs with the aim of obtaining precise estimates of population characteristics. For the purposes of our research, our final sample size of 1164 also provides a high degree of precision and statistical power. For instance, at alpha $=$ 
.05 , it provides $92 \%$ and $99.9 \%$ power to detect population correlations of .10 and .15 , respectively.

\subsection{Materials}

\subsubsection{Problematic Smartphone Use}

Problematic smartphone use was measured using a 7-item scale (see item text in the results section). Items were assessed on a 5-point Likert scale: Strongly disagree, Disagree, Somewhat agree, Agree, Strongly agree. Scale scores were the mean response. The primary focus of this research was on the problematic smartphone use scale. Problematic smartphone use items were selected from two validated problematic smartphone usage scales, the Adolescent Preoccupation with Screens Scale (APSS, Hunter et al., 2017) adapted for adults (Horwood \& Anglim, 2020a), and the Mobile Phone Problem Use Scale (MPPUS; Bianchi \& Phillips, 2005). The items were selected based on their published validity in previous research and their capacity to provide broad coverage of the construct of problematic smartphone use (Horwood \& Anglim, 2018, 2019). In a separate young adult sample $(n=1,888)$ the correlations of the current scale with MPPUS $(r=.87)$ and APSS $(r$ $=.87$ ) was high and similar to the correlation between the MPPUS and the APSS ( $r$ $=.88)$. Cronbach's alpha was 0.89 .

\subsubsection{Technology Concern}

The construct of technology concern was developed to explore the concept of a generalized fear of the potential harms of new technology. As there were no published and validated scales to the authors knowledge, technology concern was measured using a 4-item scale developed by the authors and the program producer (Table 1). The response scale was the same as for problematic smartphone use. Cronbach's alpha was 0.73 . A factor analysis of the problematic smartphone use and technology concern items is presented in the online supplement. There was strong support for the proposed two-factor solution.

\subsubsection{Self-Rated Daily Smartphone Use}

Participants provided a self-rated estimate of their average daily smartphone use. Before accessing their objective usage, participants were asked the following question: "How much time do you think you spend on your smartphone every day?" Response options were: "Less than 1 hour", "1 to 2 hours", "2 to 3 hours", "3 to 4 hours", "More than 4 hours". There was also an "Unsure" option, but as previously mentioned, participants who provided this response were excluded from the study; only 7 participants were excluded based solely on this exclusion criteria, and this response was uncorrelated with age, gender, PSU, and technology concerns. In order to provide a more meaningful comparison with objective daily use, we converted response that estimated average minutes per day of usage. For the first four categories, we coded it as the mid-point between response options: i.e., "Less than 1 hour" was 30 minutes, "1 to 2 hours" was 90 minutes, "2 to 3 hours" was 150 minutes, "3 to 4 hours" was 210 minutes. We coded "More than 4 hours" as 357 minutes, which was the mean amount of time reported for objective daily smartphone use amongst those with 4 or more hours of objective usage. 


\subsubsection{Objective Daily Smartphone Use}

Objective smartphone use was the average minutes per day of screen time in the preceding week. Both iPhone and Android users were provided with video instructions on how to access their respective Screen Time and Digital Wellbeing tools. In the case of iPhone users, they were instructed to access Screen Time and go to the preceding week (i.e., where a full 7 days of data were available) and record the average daily hours and minutes of screen time. In the case of Android users, they recorded the hours and minutes of usage over the last 7 full days. We then computed average daily usage as the average of these 7 provided days of usage.

\section{Results}

\subsection{Smartphone Use}

Table 1 presents the percentage agreeing with each item (i.e., agree or strongly agree) and Table 2 presents descriptive statistics and correlations for the key variables in the study. Overall, the results suggest a sizable proportion of the sample endorse problematic smartphone use items and that most people have concerns about technology. For example, around a third of the sample felt that they spent too much time on their smartphone and that they found it difficult to switch off. Around two-thirds of the sample had concerns about the impact of excessive smartphone use on people's well-being and children.

Mean objective smartphone use was 184 minutes per day $(S D=132)$. Average minutes per day of smartphone screen time for various percentiles were 37 (10th), 69 (20th), 95 (30th), 129 (40th), 157 (50th), 193 (60th), 230 (70th), 284 (80th), and 372 (90th). Self-rated smartphone use was highly correlated with objective smartphone use $(r=.73)$. However, assuming objective ratings represent the truth, people tended to underestimate their smartphone use. Mean self-ratings were only $85 \%$ of mean objective values. When using the hour-long ranges of the self-ratings, $56 \%$ of self-ratings were accurate, $13 \%$ were overestimates, and $31 \%$ were underestimates.

Problematic smartphone use had a strong correlation with both objective ( $r$ $=.50)$ and self-rated smartphone use $(r=.59)$. This correlation with self-rated smartphone use was significantly larger $(p<.0001)$ than the correlation with objective use. Technology concerns were unrelated to smartphone use or age.

\subsection{Age and Smartphone Use}

In order to examine the effect of age on objective use, self-rated use, and problematic smartphone use, correlations (Table 2), group differences (Table 3), and regression models were examined (Table 4). Regression models included gender $(0$ $=$ Male, $1=$ Female) as well as linear and quadratic effects of age. Age had a strong negative correlation with objective $(r=-.44)$, self-rated $(r=-.52)$ and problematic $(r=-.51)$ smartphone use. The correlation of age with objective use was significantly less than age with self-rated use $(p<.0001)$. In the case of objective and self-rated use, the effect of age was linear with no significant quadratic effect. For instance, the model of objective smartphone use suggests that for every 20 years older a person is, they use their smartphone for 70 minutes less per day. This effect of age can also be seen by examining the categorical age group means in Table 3, whereby the youngest age group (18 to 24 ) is averaging 255 minutes per day on their 
smartphone and the oldest $(65+)$ is averaging 99 minutes per day. In contrast, problematic smartphone use was not entirely linear. An examination of a scatterplot and as implied by Table 3 and the age-squared coefficient in Table 4, problematic smartphone use was high and relatively similar from ages 18 to 35 and then gradually begins to decline, where the decline gets more rapid after about age 40 . Overall, age and gender accounted for $20 \%$ of objective use, $28 \%$ of self-rated use, and $26 \%$ of problematic use.

For completeness, we include an assessment of the role of gender in smartphone use. Females used their smartphone slightly more than males based on both self-rating and objective measures. In terms of objective use, females spent on average $22 \%$ more time on their smartphone than males. This amounted to an average of 36 more minutes more per day. Similar but slightly smaller estimates of gender differences were obtained using self-ratings. In contrast, gender differences in problematic smartphone use were relatively smaller. It is also worth noting that after controlling for age in the regression models, the effect of gender on self-rated smartphone use (but not objective use) was no longer statistically significant.

\section{Discussion}

The current study utilized a large nationally representative sample to examine (a) the effect of age on smartphone use, (b) the degree to which people are able to accurately estimate their amount of smartphone use, and (c) the degree to which problematic smartphone use could be explained by age, amount of smartphone use, and technology concern. Several interesting findings emerged. First, there was a linear effect of age on objective and self-rated smartphone use, while problematic use was relatively high and stable from ages 18 to 35 , before a rapid decline from around age 40. Second, while the correlation between problematic smartphone use and amount of use was high, they were far from equivalent. Third, self-rated smartphone use correlated highly with objective use, although people did tend to underestimate their smartphone use. Fourth, despite a high degree technology concern, this was unrelated to amount of smartphone use and problematic smartphone use.

\subsection{Problematic Smartphone Use and Amount of Smartphone Use}

Overall self-rated problematic smartphone use in the present sample was quite high. Of the total sample, $35 \%$ reported feeling anxious if they could not access their smartphone, almost half reported that their time spent online has increased over the past 12 months, and 37\% reported that they believe they spend too much time on their smartphone. The median amount of smartphone screen time was around two and half hours per day. These results are consistent with advances in smartphone technology resulting in more of people's daily activities being subsumed or mediated by smartphones. Nonetheless, it is interesting to consider why people report high levels of problematic use but remain heavy users of their smartphones. It is likely that the many benefits of smartphone use lead to the integration of daily routines around checking and use that people recognize as having undesirable elements. People may also be unable or unwilling to change their behavior.

Results also showed that people were reasonably accurate in estimating their amount of smartphone use. This was principally evidenced by the high correlation $(r=.73)$ between self-ratings and objective measures derived from device screen 
time settings. This estimate is substantially larger than the value obtained in past literature (Parry et al., 2020). Because the study employed a broad cross-section of ages, there was more variance in actual levels of use than is seen in studies of young people. Given that most smartphones now have screen time software pre-installed, it is likely that some, if not many, people are notified of their screen time habits either daily or weekly, which may, in part, explain the strong correlation. Nonetheless, people did slightly underestimate their amount of use, which is consistent with people partially losing track of time when using their smartphone. Results also suggest that problematic smartphone use is highly related but distinct from amount of smartphone use. Interestingly, problematic use was more strongly correlated with self-rated use than objective usage. Self-rated amount of use is reliant on the user reflecting on the things they have used their smartphone for, and how often. It is possible that on reflection users recall activities that have a salient cue attached, such as feeling guilty for wasting time or engaging in use that has drawn negative appraisal from others. Neutral activities, such as checking the weather or the news, or perhaps using utilities such as maps or flashlights are less likely to be remembered. Basing estimated smartphone use on the salience of smartphone activities that provoke a feeling or reflection would explain why, on average, people under-reported their actual (objective) usage.

\subsection{Age and Smartphone Use}

Corroborating past research with smaller and less representative samples (e.g. Alhassan et al., 2018; Csibi et al., 2019; Mitchell \& Hussain, 2018; Van Deursen et al., 2015), the present study provides a nuanced understanding of agerelated effects on smartphone use. In our large nationally representative sample, we found that the amount of time spent using a smartphone, whether measured objectively or using self-ratings, declined linearly with age, while problematic use was relatively stable over young adulthood before declining rapidly from around age 40. Thus, young adults (e.g., 18 to 25) typically perceived their high levels of smartphone use as less problematic than slightly older adults with an equivalent amount of use. Presumably, developmental and generational effects can be used to explain these results.

First, the observed age-related differences in smartphone use and problematic smartphone use may be explained by biological processes and maturational norms. Social clock theory describes the natural tendency of people to internalize social norms around age-appropriate development (Neugarten, 1979). That is, most people follow a social timeline whereby major life events, such as getting married, having children, or retiring, typically occur at predictable ages. The youngest age group in the present study, people aged 18-25, may be more inclined to use smartphones at a higher rate due to their limited social responsibilities at that age. Younger people are less likely to have an established career to tend to, a family to be responsible for, or elderly parents that need care. Instead, they are more likely to be engaging in high levels of socializing and exploration of their world as young adults. As people age, social clock theory suggests that more and more timeconsuming encumbrances are embraced, perhaps leaving less time for 'frivolous' smartphone activities such as games, social media, or watching video content. If biological age has an effect on smartphone use, the current 18-25 year olds would 
be expected to use their smartphone less as they age in line with the current crosssectional results.

Second, age-related difference can also be understood in terms of generational change. Younger people are more likely to have encountered smartphone technology from a younger and more impressionable age than older people (i.e. the current cohort of 18-25 year olds would have been 8-15 years old when modern smartphones were first introduced). The idea of digital nativity is that young people who have not known a world without the internet and associated internet-enabled devices are somehow more attuned and enmeshed with technology than those who can remember the pre-internet world (Prensky, 2001). Although there are some studies that have reported higher internet-enabled technology use (and higher problematic internet use) by digital natives, other studies have found that younger people are less competent with IT-related tasks than older people (Calvani, Fini, Ranieri, \& Picci, 2012; Guo, Dobson, \& Petrina, 2008). One explanation for why younger people are heavier users of smartphones than older people is that younger people see smartphones as an integral part of day-to-day life, in much the same way that older people might consider using electricity or driving around in cars. Younger people may be more willing and adept at integrating technology into their lives because, for them, it has always been there. If the effect of age on smartphone use is a cohort effect, longitudinal analyses should reflect little to no decrease in usage as the current cohort of 18-25 year olds age into their $30 \mathrm{~s}$ and beyond.

The results also indicated that problematic smartphone use was relatively stable in young adulthood and did not begin declining until around age 40. These findings are less intuitive to explain considering the strong association between amount of smartphone use and perceptions of problematic use. It may be that that age is also related to perceptions of what is an appropriate amount of use. For instance, younger adults may use their smartphone more than older adults but they may see higher levels of use as more normatively appropriate. In addition, young adults typically have more discretionary time than adults in their 30 s and 40 s who may have more work and family commitments. Adults with large work and family commitments may experience more problems if they spend large amounts of time on their smartphone.

\subsection{Technology Concern}

The results of the present study indicate a high level of technology concerns within the sample. Over two thirds of the sample believed that overuse of smartphones can negatively impact wellbeing, three quarters had concerns about their personal data, and over $70 \%$ believed that mobile devices can have a negative impact on children. Despite these concerns, people still had high levels of smartphone use and these concerns were relatively unrelated to amount of smartphone use and problematic smartphone use. Furthermore, there was no effect of age on technology concerns. As such, stereotypes that older people are more suspicious of technology than younger people appear to be unfounded.

It is likely that the relationship between technology concerns and technology behavior is complex. The present study considered the argument that problematic smartphone use merely reflects cyclical technology concerns in disguise. If this were 
the case, we might expect to see strong correlations between technology concerns and problematic use. However, our data indicated that there was no relationship between the two. On the other hand, it could be reasonable to expect that as a person's fear of technology use increases, their use of technology would decline, however as there was no evidence of any relationship between technology beliefs and the behaviors, this argument also fails to hold. One explanation for why there is no relationship between beliefs and behavior is that people can hold incongruous beliefs about technology. They can believe that technology use is harmful in general terms, but that their own technology use will not harm them. The results do suggest, however, that the cyclical social technology fears believed to be at the heart of problematic smartphone use are unrelated to the construct. That is, while people may genuinely and predictably fear that new technology is harmful, to young people in particular, it is not an explanation for the growing evidence that problematic smartphone use is a genuine psychological construct (Horwood \& Anglim, 2020b). It also suggests that problematic use has more to do with self-appraisal and experience of smartphone use than actual time spent on the device.

\subsection{Limitations}

Several limitations should be noted. First, because data collection took place during the coronavirus pandemic, it is worth considering what, if any, effect this may have on the generalizability of the results. To the extent that people were out of work or confined to their homes, this may have led to elevated levels of smartphone use. It is worth noting that the timing of data collection (i.e., 11th of June, 2020) and the seven days before (i.e., from which the average weekly usage data was obtained) was a period characterized by almost no community spread of coronavirus in Australia. In broad terms, in April, Australia engaged in a mild form of lockdown where people could leave their home only for work/study (if they couldn't work from home), exercise, shopping, and caring. This was followed by May and early June which were characterized by a loosening of many restrictions, particularly in states that were COVID-free (for further discussion of the context, see Anglim \& Horwood, 2020).

Second, the measurement of objective smartphone screen time includes various challenges. Using built-in screen time data relies on participants accurately looking up and recording this data. To facilitate this task, we provided short navigation videos for both $\mathrm{iOS}$ and Android systems. Also, there may be inconsistencies in how devices record screen time. There is the issue of whether a screen time average reflects only the owner's usage or whether it includes additional usage by another person such as a partner or child. Third, the data were not able to shed light on the types of activities that people of different ages used their smartphones for. This information would be useful to collect in future studies of problematic smartphone use. Fourth, many people spread their screen time across more than one device, typically a smartphone and a tablet, and depending on the settings in each device, some iOS screen time averages could be for only the users smartphone, while others could be for their iOS smartphone and iOS tablet.

Finally, our categorization of self-reported smartphone use (i.e., 'less than 1 hour', ' 1 to 2 hours' etc.) necessarily constricted participants ability to enter any estimation they wished, and likely reduced the granularity of the data. Future 
research could employ more nuanced measures of usage and examine the effect of different measurement approaches on the accuracy of self-report estimates.

\subsection{Conclusion}

The current study contributes to the overall understanding of the relationship between age and smartphone use. A major contribution to the literature is the large, nationally representative sample of adults that enabled a comprehensive assessment of the effect of age on self-rated and objective smartphone use. There was a strong linear effect of age on self-rated and objective smartphone use, while problematic smartphone use was relatively stable in young adulthood before declining rapidly around age 40. Furthermore, the research contributes to the emerging debate about whether smartphones and related technologies are potentially harmful to young people or simply the latest manifestation of predictable concerns about new technology. The results indicate that people have concerns about new technology, but they are unrelated to the degree to which they use their smartphones. It is likely that problematic smartphone use is a complex psychological construct underpinned by subjective experience and individual differences beyond amount of use.

\section{References}

Alhassan, A. A., Alqadhib, E. M., Taha, N. W., Alahmari, R. A., Salam, M., \& Almutairi, A. F. (2018). The relationship between addiction to smartphone usage and depression among adults: a cross sectional study. BMC Psychiatry, $18(1), 148$. doi:10.1186/s12888-018-1745-4

Alter, A. (2017). Irresistible: The rise of addictive technology and the business of keeping us hooked: Penguin.

Anderson, J., \& Rainie, L. (2018). The Future of Well-Being in a Tech-Saturated World. Retrieved from https://www.pewresearch.org/internet/2018/04/17/the-future-of-well-being-in-a-tech-saturated-world/

Anglim, J., \& Horwood, S. (2020). Effect of the COVID-19 Pandemic and Big Five Personality on Subjective and Psychological Well-Being. Social Psychological and Personality Science, 1948550620983047.

Belson, W. A., \& Belson, W. A. (1978). Television violence and the adolescent boy.

Bianchi, A., \& Phillips, J. G. (2005). Psychological predictors of problem mobile phone use. CyberPsychology \& Behavior, 8(1), 39-51. doi:doi.org/10.1089/cpb.2005.8.39

Bickham, D. S., \& Rich, M. (2006). Is television viewing associated with social isolation?: roles of exposure time, viewing context, and violent content. Archives of pediatrics \& adolescent medicine, 160(4), 387-392. doi:doi:10.1001/archpedi.160.4.387

Biddle, S. J., Gorely, T., \& Marshall, S. J. (2009). Is television viewing a suitable marker of sedentary behavior in young people? Annals of behavioral medicine, 38(2), 147-153. doi:doi.org/10.1007/s12160-009-9136-1

Busch, P. A., \& McCarthy, S. (2020). Antecedents and consequences of problematic smartphone use: A systematic literature review of an emerging research area. Computers in Human Behavior, 106414. doi:doi.org/10.1016/j.chb.2020.106414

Calvani, A., Fini, A., Ranieri, M., \& Picci, P. (2012). Are young generations in secondary school digitally competent? A study on Italian teenagers. Computers \& Education, 58(2), 797-807. doi:https://doi.org/10.1016/j.compedu.2011.10.004

Carnagey, N. L., Anderson, C. A., \& Bushman, B. J. (2007). The effect of video game violence on physiological desensitization to real-life violence. Journal of experimental social psychology, 43(3), 489-496. doi:doi:10.1016/j.jesp.2006.05.003

Clement, J., \& Miles, M. (2018). Screen schooled: Two veteran teachers expose how technology overuse is making our kids dumber: Black Inc.

Csibi, S., Griffiths, M. D., Demetrovics, Z., \& Szabo, A. (2019). Analysis of Problematic Smartphone Use Across Different Age Groups within the 'Components Model of Addiction'. International Journal of Mental Health and Addiction. doi:10.1007/s11469-019-00095-0

Dewandre, N. (2020). Big Data: From modern fears to enlightened and vigilant embrace of new beginnings. Big Data \& Society, 7(2). doi:doi.org/10.1177/2053951720936708

Ellis, D. A., Davidson, B. I., Shaw, H., \& Geyer, K. (2019). Do smartphone usage scales predict behavior? International Journal of Human-Computer Studies, 130, 86-92. doi:doi.org/10.1016/j.ijhcs.2019.05.004

Ford, E. S., Li, C., Zhao, G., Pearson, W. S., Tsai, J., \& Churilla, J. R. (2010). Sedentary behavior, physical activity, and concentrations of insulin among US adults. Metabolism, 59(9), 1268-1275. doi:doi.org/10.1016/j.metabol.2009.11.020

Guo, R. X., Dobson, T., \& Petrina, S. (2008). Digital Natives, Digital Immigrants: An Analysis of Age and Ict Competency in Teacher Education. Journal of Educational Computing Research, 38(3), 235-254. doi:10.2190/EC.38.3.a

Horwood, S., \& Anglim, J. (2018). Personality and problematic smartphone use: A facet-level analysis using the Five Factor Model and HEXACO frameworks. Computers in Human Behavior, 85, 349-359. doi:doi.org/10.1016/j.chb.2018.04.013

Horwood, S., \& Anglim, J. (2019). Problematic smartphone usage and subjective and psychological well-being. Computers in Human Behavior, 97, 44-50. doi:10.1016/j.chb.2019.02.028 
Horwood, S., \& Anglim, J. (2020a). Emotion Regulation Difficulties, Personality, and Problematic Smartphone Use. Cyberpsychology, Behavior, and Social Networking.

Horwood, S., \& Anglim, J. (2020b). Self and Other Ratings of Problematic Smartphone Use: The Role of Personality and Relationship Type. Computers in Human Behavior, 116, 106634. doi:doi.org/10.1016/j.chb.2020.106634

Hsieh, H.-F., Hsu, H.-T., Lin, P.-C., Yang, Y.-J., Huang, Y.-T., Ko, C.-H., \& Wang, H.-H. (2020). The Effect of Age, Gender, and Job on Skin Conductance Response among Smartphone Users Who are Prohibited from Using Their Smartphone. International journal of environmental research and public health, 17(7). doi:10.3390/ijerph17072313

Hunter, S. C., Houghton, S., Zadow, C., Rosenberg, M., Wood, L., Shilton, T., \& Lawrence, D. (2017). Development of the Adolescent Preoccupation with Screens Scale. BMC public health, 17(1), 652. doi:https://doi.org/10.1186/s12889-0174657-1

Kirsh, S. J. (2003). The effects of violent video games on adolescents: The overlooked influence of development. Aggression and violent behavior, 8(4), 377-389. doi:doi.org/10.1016/S1359-1789(02)00056-3

Kremar, M., \& Greene, K. (1999). Predicting exposure to and uses of television violence. Journal of communication, 49(3), 24-45. doi:doi.org/10.1111/j.1460-2466.1999.tb02803.x

Lee, M., Han, M., \& Pak, J. (2018). Analysis of Behavioral Characteristics of Smartphone Addiction Using Data Mining. Applied Sciences, 8(7), 1191. doi:doi.org/10.3390/app8071191

Mitchell, L., \& Hussain, Z. (2018). Predictors of Problematic Smartphone Use: An Examination of the Integrative Pathways Model and the Role of Age, Gender, Impulsiveness, Excessive Reassurance Seeking, Extraversion, and Depression. Behavioral sciences (Basel, Switzerland), 8(8). doi:10.3390/bs8080074

Montag, C., \& Elhai, J. D. (2020). Discussing digital technology overuse in children and adolescents during the COVID-19 pandemic and beyond: On the importance of considering Affective Neuroscience Theory. Addictive Behaviors Reports, 12. doi:doi.org/10.1016/j.abrep.2020.100313

Montag, C., \& Walla, P. (2016). Carpe diem instead of losing your social mind: Beyond digital addiction and why we all suffer from digital overuse. Cogent Psychology, 3(1). doi:doi.org/10.1080/23311908.2016.1157281

Neugarten, B. (1979). Time, age, and the life cycle. American Journal of Psychiatry, 136(7), 887-894. doi:10.1176/ajp.136.7.887

Orben, A. (2020). The Sisyphean Cycle of Technology Panics. Perspectives on Psychological Science., 15 (5), $1143-1157$. doi:10.1177/1745691620919372

Pan, Y.-C., Lin, H.-H., Chiu, Y.-C., Lin, S.-H., \& Lin, Y.-H. (2019). Temporal stability of smartphone use data: Determining fundamental time unit and independent cycle. JMIR mHealth and uHealth, 7(3), e12171. doi:doi:10.2196/12171

Parry, D. A., Davidson, B. I., Sewall, C., Fisher, J. T., Mieczkowski, H., \& Quintana, D. (2020). Measurement Discrepancies Between Logged and Self-Reported Digital Media Use: A Systematic Review and Meta-Analysis.

Prasad, S., Harshe, D., Kaur, N., Jangannavar, S., Srivastava, A., Achanta, U., . . Harshe, G. (2018). A Study of Magnitude and Psychological Correlates of Smartphone Use in Medical Students: A Pilot Study with A Novel Telemetric Approach. Indian Journal of Psychological Medicine, 40(5), 468-475. doi:10.4103/ijpsym.Ijpsym_133_18

Prensky, M. (2001). Digital Natives, Digital Immigrants Part 1. On the Horizon, 9(5), 1-6. doi:10.1108/10748120110424816

Research, P. (2019). Mobile Factsheet. Retrieved from Washington, DC: https://www.pewresearch.org/internet/fact-sheet/mobile/

Rey-López, J. P., Vicente-Rodríguez, G., Biosca, M., \& Moreno, L. A. (2008). Sedentary behaviour and obesity development in children and adolescents. Nutrition, metabolism and cardiovascular diseases, 18(3), $242-251$. doi:doi.org/10.1016/j.numecd.2007.07.008

Rozgonjuk, D., Levine, J. C., Hall, B. J., \& Elhai, J. D. (2018). The association between problematic smartphone use, depression and anxiety symptom severity, and objectively measured smartphone use over one week. Computers in Human Behavior, 87, 10-17. doi:doi.org/10.1016/j.chb.2018.05.019

Rozgonjuk, D., Sindermann, C., Elhai, J. D., \& Montag, C. (2020). Fear of Missing Out (FoMO) and social media's impact on daily-life and productivity at work: Do WhatsApp, Facebook, Instagram, and Snapchat Use Disorders mediate that association? Addictive Behaviors, 110, 106487.

Ryding, F. C., \& Kuss, D. J. (2020). Passive objective measures in the assessment of problematic smartphone use: A systematic review. Addictive Behaviors Reports, 11. doi:10.1016/j.abrep.2020.100257

Stern, S. E. (1999). Addiction to technologies: A social psychological perspective of Internet addiction. CyberPsychology \& Behavior, 2(5), 419-424. doi:doi-org.ezproxy-f.deakin.edu.au/10.1089/cpb.1999.2.419

Van Deursen, A. J., Bolle, C. L., Hegner, S. M., \& Kommers, P. A. (2015). Modeling habitual and addictive smartphone behavior: The role of smartphone usage types, emotional intelligence, social stress, self-regulation, age, and gender. Computers in Human Behavior, 45, 411-420. doi:doi.org/10.1016/j.chb.2014.12.039

Wang, H.-Y., Sigerson, L., \& Cheng, C. (2019). Digital nativity and information technology addiction: Age cohort versus individual difference approaches. Computers in Human Behavior, 90, 1-9. doi:10.1016/j.chb.2018.08.031

Wilcockson, T. D., Ellis, D. A., \& Shaw, H. (2018). Determining typical smartphone usage: What data do we need? Cyberpsychology, Behavior, and Social Networking, 21(6), 395-398. doi:doi.org/10.1089/cyber.2017.0652

Zhitomirsky-Geffet, M., \& Blau, M. (2016). Cross-generational analysis of predictive factors of addictive behavior in smartphone usage. Computers in Human Behavior, 64, 682-693. doi:doi.org/10.1016/j.chb.2016.07.061 
Table 1

Item Endorsement and Correlations

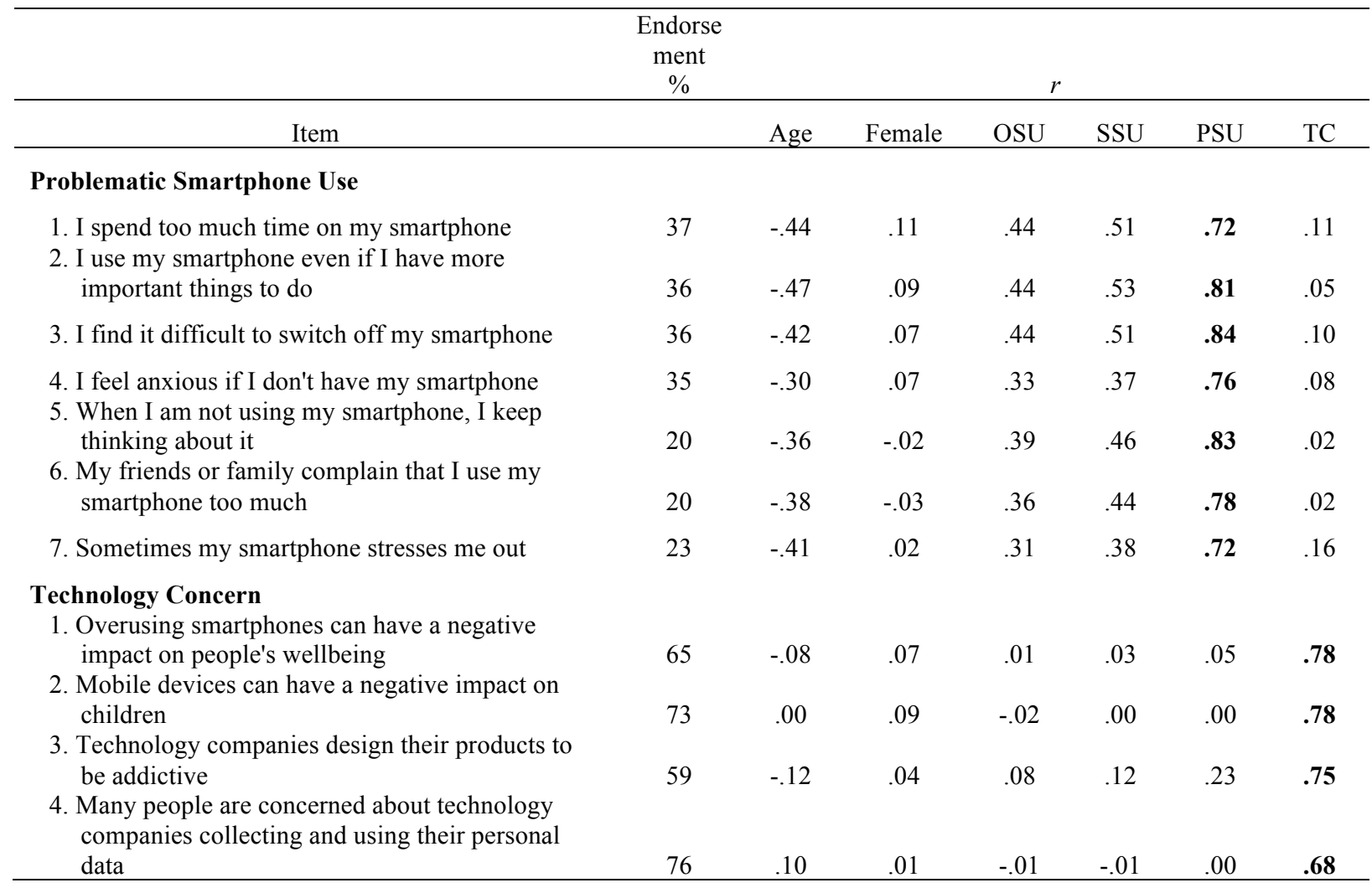

Note. OSU = Objective Smartphone Use, SSU = Self-Rated Smartphone Use, PSU =

Problematic Smartphone Use, TC $=$ Technology Concern. Female is code $0=$ Male, $1=$ Female. Percentage endorsement (\%) is the percentage of responses indicating Agree or Strongly Agree. Columns $r$ are the correlation between the item and the variables. Item-scale total correlations are in bold.

Table 2

Descriptive Statistics and Correlation Matrix

\begin{tabular}{|c|c|c|c|c|c|c|c|}
\hline Variable & $M$ & $S D$ & 1 & 2 & 3 & 4 & 5 \\
\hline 1. Age & 44.89 & 16.34 & & & & & \\
\hline 2. Female & 0.51 & 0.50 & $-.14 * * *$ & & & & \\
\hline 3 Objective Smartphone Use & 183.84 & 131.61 & $-.44 * * *$ & $.13 * * *$ & & & \\
\hline 4. Self-Rated Smartphone Use & 156.05 & 112.91 & $-.52 * * *$ & $.12 * * *$ & $.73 * * *$ & & \\
\hline 5. Problematic Smartphone Use & 2.72 & 0.94 & $-.51 * * *$ & $.06 *$ & $.50 * * *$ & $.59 * * *$ & \\
\hline 6. Technology Concern & 3.89 & 0.71 & -.04 & $.07 *$ & .02 & .05 & $.10 * * *$ \\
\hline
\end{tabular}

Note. Female is coded $0=$ Male, $1=$ Female. Absolute correlations greater than or equal to .06, .08 , and .10 are statistically significant at $.05, .01$, and .001 respectively.

$* p<.05 ; * * p<.01 ; * * * p<.001$ 
Table 3

Age and Gender Differences in Smartphone Use

\begin{tabular}{lccccccc} 
& & \multicolumn{2}{c}{$\begin{array}{c}\text { Objective } \\
\text { Smartphone Use }\end{array}$} & \multicolumn{2}{c}{$\begin{array}{c}\text { Self-Rated } \\
\text { Smartphone Use }\end{array}$} & \multicolumn{2}{c}{$\begin{array}{c}\text { Problematic } \\
\text { Smartphone Use }\end{array}$} \\
\cline { 3 - 8 } Group & $n$ & $M$ & $S D$ & $M$ & $S D$ & $M$ & $S D$ \\
\hline $\begin{array}{l}\text { Overall } \\
\text { Gender }\end{array}$ & 1164 & 184 & 132 & 156 & 113 & 2.72 & 0.94 \\
$\quad$ Male & 571 & 165 & 127 & 142 & 108 & 2.66 & 0.99 \\
$\quad$ Female & 590 & 201 & 133 & 170 & 116 & 2.77 & 0.89 \\
Age & & & & & & & \\
18 to 24 & 156 & 255 & 143 & 231 & 109 & 3.20 & 0.73 \\
25 to 34 & 219 & 251 & 139 & 223 & 113 & 3.20 & 0.84 \\
35 to 44 & 220 & 206 & 115 & 179 & 99 & 3.10 & 0.88 \\
45 to 54 & 207 & 167 & 114 & 132 & 99 & 2.58 & 0.84 \\
55 to 64 & 182 & 119 & 104 & 99 & 87 & 2.21 & 0.83 \\
65+ & 180 & 99 & 83 & 67 & 55 & 1.92 & 0.63 \\
\hline
\end{tabular}

Note. Objective and self-rated smartphone use index average daily minutes of screen time.

Table 4

Regression Models Predicting Smartphone Use

\begin{tabular}{lccc}
\hline & $\begin{array}{c}\text { Objective } \\
\text { Smartphone Use }\end{array}$ & $\begin{array}{c}\text { Self-Rated } \\
\text { Smartphone Use } \\
B(\mathrm{se})\end{array}$ & $\begin{array}{c}\text { Problematic } \\
\text { Smartphone Use } \\
(\mathrm{se})\end{array}$ \\
\hline Predictors & & & \\
Intercept & $171.16^{* * *}(6.08)$ & $147.69 * * *(4.96)$ & $2.80^{* * *}(0.04)$ \\
Age-centered & $-70.11^{* * *}(4.34)$ & $-72.06^{* * *}(3.56)$ & $-0.57^{* * *}(0.03)$ \\
Age-squared & $3.58(5.33)$ & $3.46(4.35)$ & $-0.10^{* *}(0.04)$ \\
Female & $19.52^{* *}(6.97)$ & $11.15(5.69)$ & $-0.03(0.05)$ \\
Residual se & 97.39 & & 0.81 \\
Adjusted $\mathrm{R}^{2}$ & $.20^{* * *}$ & 96.07 & $.26^{* * *}$ \\
\hline
\end{tabular}

Note. Age-centered $=($ Age -45$) / 20$. Age-squared $=($ Age-Centred $) *($ Age-Centred $)$. Female is coded $0=$ Male, $1=$ Female. I.e., Intercept equates to estimated score on the outcome variable for 45-year old male. Age-centered parameter equates to predicted increase in outcome variable for a 20 -year increase in age.

$* p<.05 ; * * p<.01 ; * * * p<.001$ 


\section{Online Supplement}

\subsection{Analyses of Entire Valid Sample $(n=1790)$}

As a robustness check, below we report the results of the current study on the full $n=1790$ sample (i.e., cases were excluded only for failing validity checks, but not because they were missing objective or self-rated usage). Where responses to particular variables were missing, pairwise deletion was employed.

\section{Table S1}

Item Endorsement and Correlations using full sample $(n=1790)$ and Pairwise Deletion.

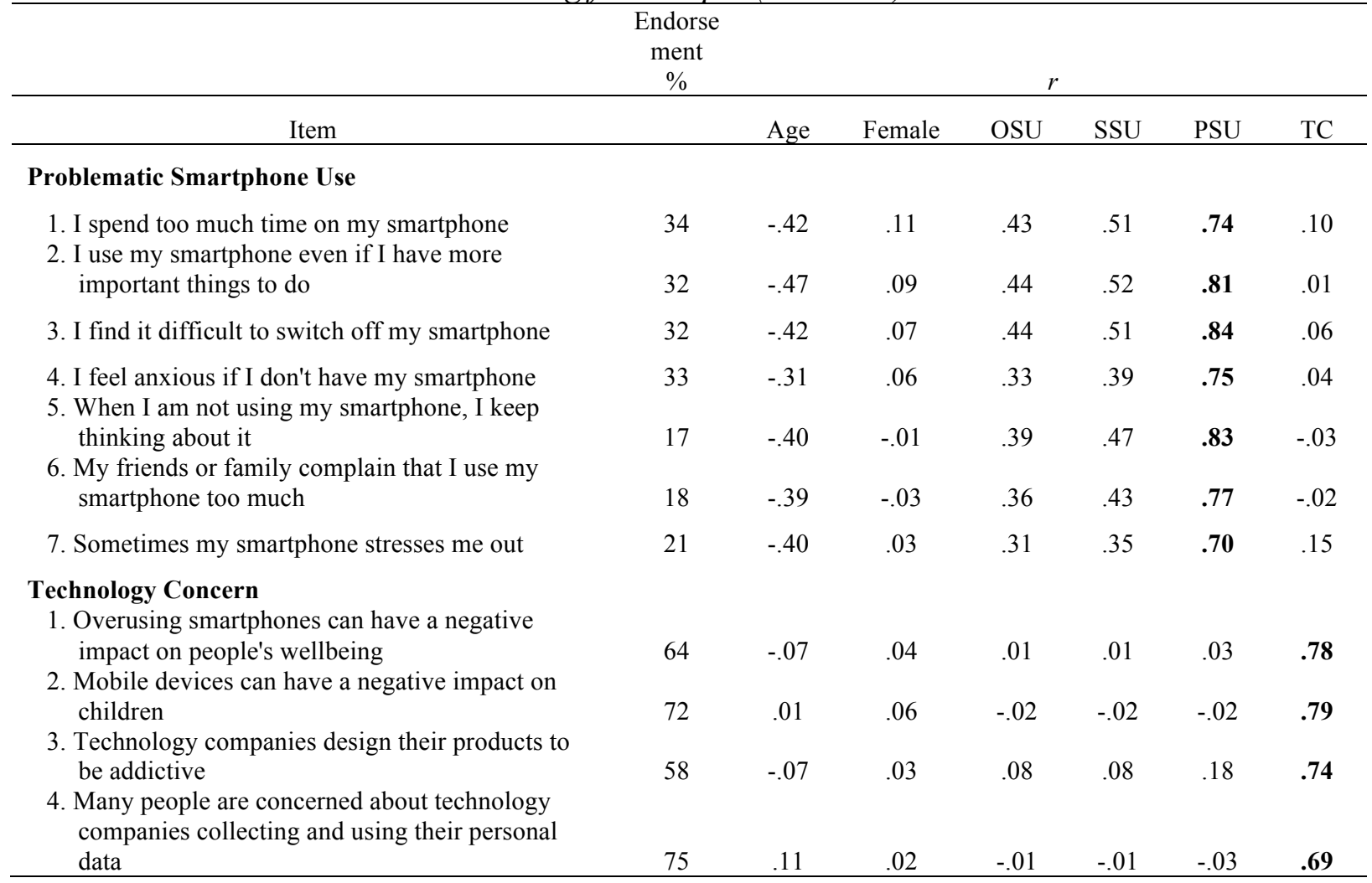

Note. OSU = Objective Smartphone Use, SSU = Self-Rated Smartphone Use, PSU = Problematic Smartphone Use, TC $=$ Technology Concern. Female is code $0=$ Male, $1=$ Female. Percentage endorsement (\%) is the percentage of responses indicating Agree or Strongly Agree. Columns $r$ are the correlation between the item and the variables. Item-scale total correlations are in bold. 


\section{Table S2}

Descriptive Statistics and Correlation Matrix using full sample $(n=1790)$ and Pairwise Deletion.

\begin{tabular}{lrrccccc}
\hline \multicolumn{1}{c}{ Variable } & \multicolumn{1}{c}{$M$} & \multicolumn{1}{c}{$S D$} & 1 & 2 & 3 & 4 & 5 \\
\hline 1. Age & 46.17 & 16.37 & & & & & \\
2. Female & 0.52 & 0.50 & $-.13^{* * *}$ & & & & \\
3 Objective Smartphone Use & 184.41 & 131.91 & $-.44^{* * *}$ & $.13^{* * *}$ & & & \\
4. Self-Rated Smartphone Use & 149.26 & 112.24 & $-.51^{* * *}$ & $.12^{* * *}$ & $.73^{* * *}$ & & \\
5. Problematic Smartphone Use & 2.64 & 0.92 & $-.51^{* * *}$ & $.06^{*}$ & $.49^{* * *}$ & $.58^{* * *}$ & \\
6. Technology Concern & 3.87 & 0.71 & -.01 & $.05^{*}$ & .02 & .02 & $.06^{*}$ \\
\hline
\end{tabular}

Note. Female is coded $0=$ Male, $1=$ Female. Absolute correlations greater than or equal to .05 , .07 , and .08 are statistically significant at $.05, .01$, and .001 respectively.

$* p<.05 ; * * p<.01 ; * * * p<.001$

\section{Table S3}

Age and Gender Differences in Smartphone Use using full sample $(n=1790)$ and Pairwise Deletion.

\begin{tabular}{lccccccc}
\hline & & \multicolumn{2}{c}{$\begin{array}{c}\text { Objective } \\
\text { Smartphone Use }\end{array}$} & \multicolumn{2}{c}{$\begin{array}{c}\text { Self-Rated } \\
\text { Smartphone Use }\end{array}$} & \multicolumn{2}{c}{$\begin{array}{c}\text { Problematic } \\
\text { Smartphone Use }\end{array}$} \\
\cline { 2 - 8 } Group & $n$ & $M$ & $S D$ & $M$ & $S D$ & $M$ & $S D$ \\
\hline $\begin{array}{l}\text { Overall } \\
\text { Gender }\end{array}$ & 1790 & 184 & 132 & 149 & 112 & 2.64 & 0.92 \\
$\quad$ Male & 847 & 167 & 129 & 134 & 107 & 2.59 & 0.97 \\
Female & 937 & 201 & 133 & 163 & 115 & 2.69 & 0.87 \\
Age & & & & & & & \\
18 to 24 & 204 & 255 & 143 & 232 & 111 & 3.18 & 0.74 \\
25 to 34 & 320 & 253 & 140 & 214 & 114 & 3.17 & 0.83 \\
35 to 44 & 336 & 206 & 115 & 175 & 104 & 2.98 & 0.88 \\
45 to 54 & 332 & 167 & 114 & 131 & 99 & 2.54 & 0.83 \\
55 to 64 & 284 & 121 & 103 & 100 & 85 & 2.21 & 0.75 \\
65+ & 314 & 100 & 85 & 65 & 56 & 1.90 & 0.63 \\
\hline
\end{tabular}

Note. Objective and self-rated smartphone use index average daily minutes of screen time. 


\subsection{Factor Analysis}

To assess the factor structure of the problematic smartphone and technology concern items, an exploratory factor analysis was performed. The percentage of variance explained by the first 5 unrotated factors was 39.4, 20.1, 6.8, 5.8, and 5.5. Thus, this clearly indicated two major factors. A maximum likelihood factor analysis was performed extracting two factors and using Promax rotation (see loadings in Table S5). Items clearly loaded on their theorized factor with all items loadings greater than .50 on their primary factor and no cross-loadings greater than .20 .

\section{Table S5}

Factor Loadings of Problematic Smartphone Use and Technology Concern Items

\begin{tabular}{lcc}
\multicolumn{1}{c}{ Item } & I & II \\
\hline Problematic Smartphone Use & & \\
1. I spend too much time on my smartphone & $\mathbf{. 6 5}$ & .06 \\
2. I use my smartphone even if I have more important things to do & $\mathbf{. 7 8}$ & -.04 \\
3. I find it difficult to switch off my smartphone & $\mathbf{. 8 2}$ & .01 \\
4. I feel anxious if I don't have my smartphone & $\mathbf{. 7 1}$ & .00 \\
5. When I am not using my smartphone, I keep thinking about it & $\mathbf{. 8 1}$ & -.08 \\
6. My friends or family complain that I use my smartphone too much & $\mathbf{. 7 5}$ & -.07 \\
7. Sometimes my smartphone stresses me out & $\mathbf{. 6 4}$ & .11 \\
Technology Concern & & \\
1. Overusing smartphones can have a negative impact on people's wellbeing & -.04 & $\mathbf{. 7 0}$ \\
2. Mobile devices can have a negative impact on children & -.10 & $\mathbf{. 7 3}$ \\
3. Technology companies design their products to be addictive & .15 & $\mathbf{. 6 1}$ \\
4. Many people are concerned about technology companies collecting and & & \\
using their personal data & -.07 & $\mathbf{. 5 3}$ \\
\hline
\end{tabular}

